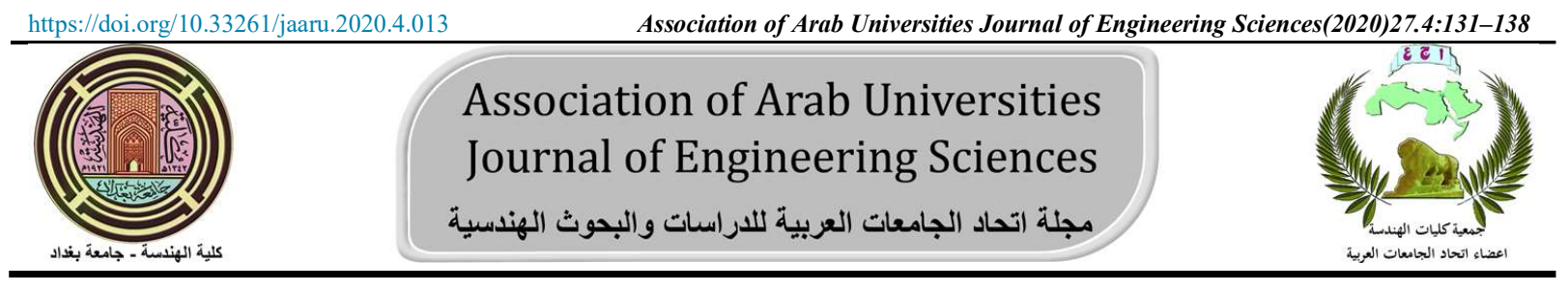

$$
\text { العنماد الجدران الترومب كتقنية مستدامة بيئًا لتحقيق كفاءة استخدام الطاقة في ابنية المناطق }
$$

\author{
عبلالله عماد علبيوي 1* ، غادة محد اسعاعيل عبل الرزاق كمونتة 2 \\ arch.abd.emad.94@gmail.com قسم هندسة العمارة،جامعة بغد/د،بغد/د،/العر/ق،
}

g.kamoona@coeng.uobaghdad.edu.iq قسم هنسة العمارة،جامعة بغد/د،بغداد،/العراق،

arch.abd.emad.94@gmail.com ، الباحث المثثل:عبداله عماد عليوي

ثر في: 31 كانون الاول 2020

الخلاصة ـ تعد الجدران الترومب (Trombe walls) احدى التقنيات المستدامة لما تمتلكه من اهميه ودور هها الفعال في الفضاءات الدات الداخلية

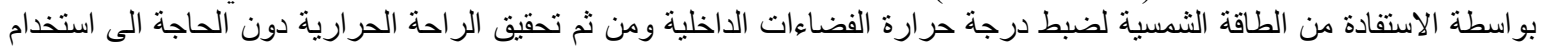

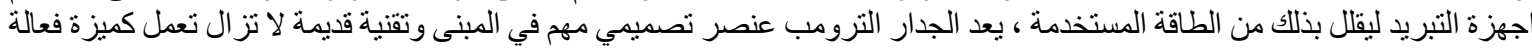

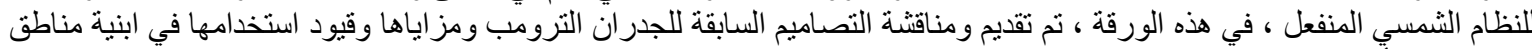

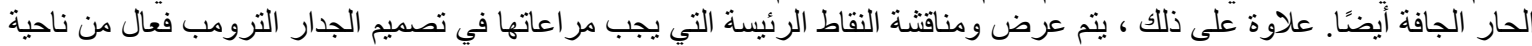

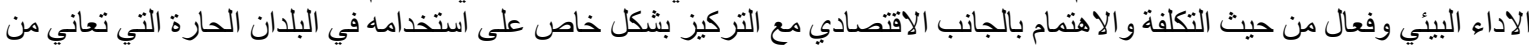

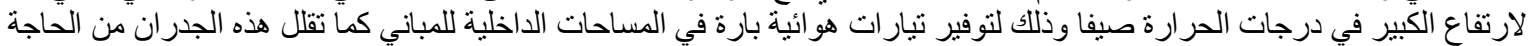

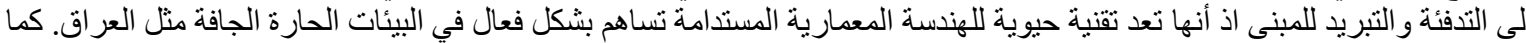

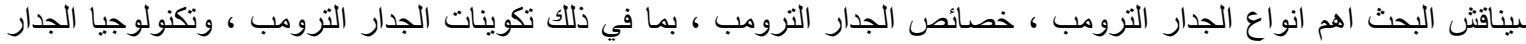
التزومب.ومدى امكانية تحقيق مفهوم كفاءة استخدام الطاقة في العراق التبان التخدام الجدار الترومب.

$$
\text { الكلمات الرئيسية: - "الاستدامة , "الراحة الحرارية , "الطاقة الثمسية , "الجدار الترومب. }
$$

الر احة الحرارية في المباني في المناطق الحارة. لهذه الاسباب يعد الجدار

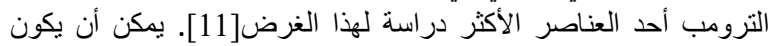

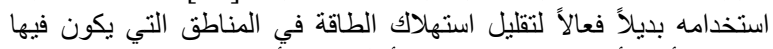

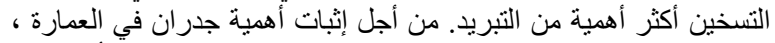

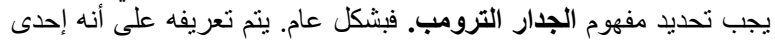

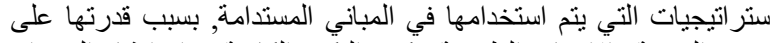

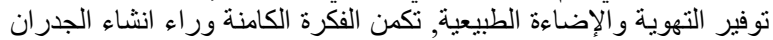

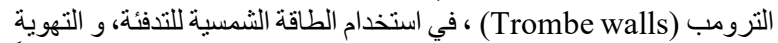

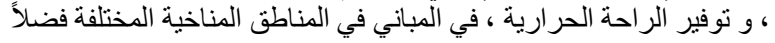

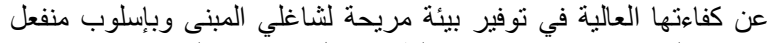

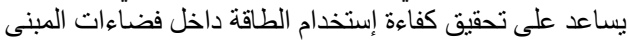

\section{2. المشكلة البحثية وهدف البحث}

عدم وجود تصور و اضح حول آلية تطبيق الجدران الترومب كو اجهة مستدامة

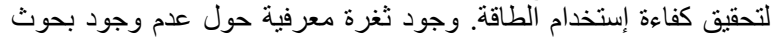

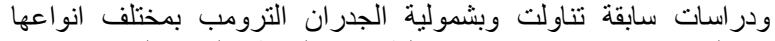

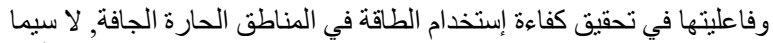

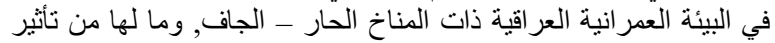
كبير في التقليل من إستهلاك الطة الطاقة. بناءاً على المشكلة البحثية تحددت الهية واهداف البحث بما يأتي :

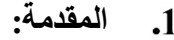

منذ زمن بعيد ، استخدم البشر جدرانًا سميكة من الطين أو الحجر لاحتجاز

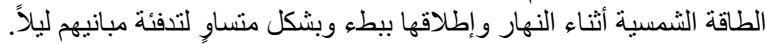

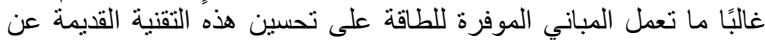

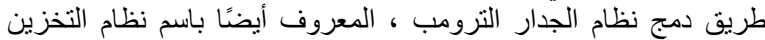

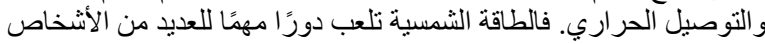

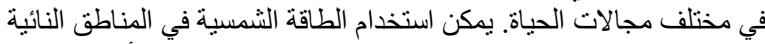

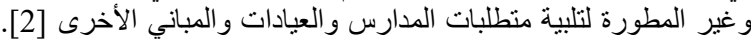

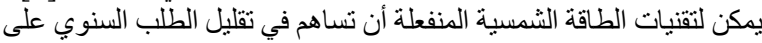

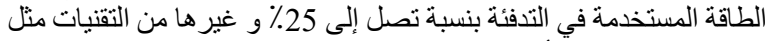

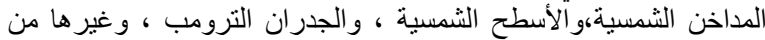

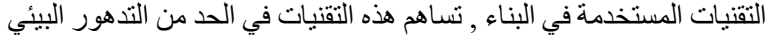

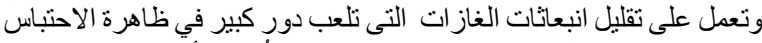

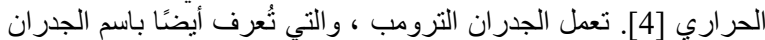

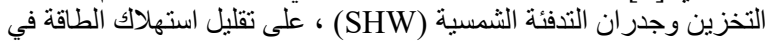

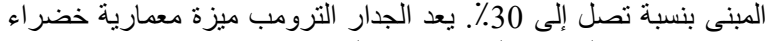

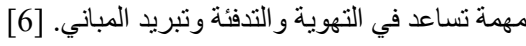

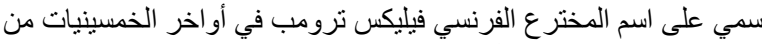

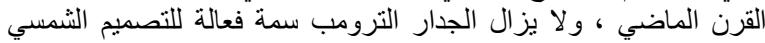

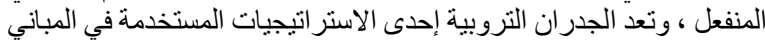

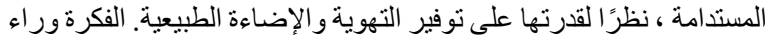
إنشاء الجدر ان الترومب هي استخدام الطاقة الثمسية للتنفئة و التهوية ولتوفئ التهير 


\section{الية عمل الجدار الترومب}

\section{.5}

تسخن الثمس الجدار،مما يتسبب في ارتفاع الهواء الساخن في الفجوة التوناء

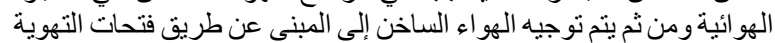

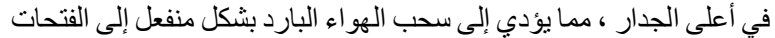

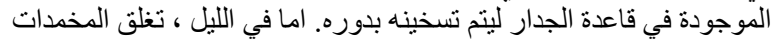

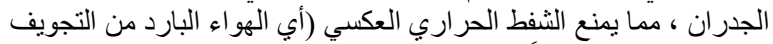

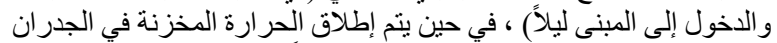

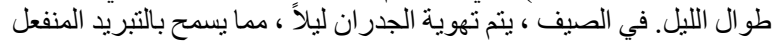

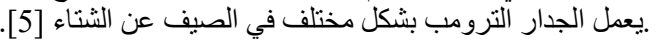

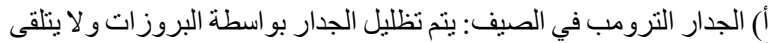

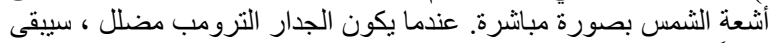

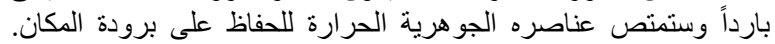

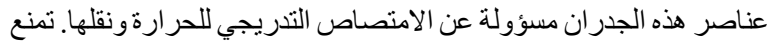

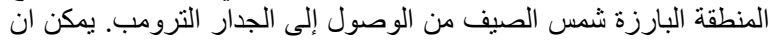
تعد مكونات الكتلة الحر ارية كبطاريات حر ارية [12].

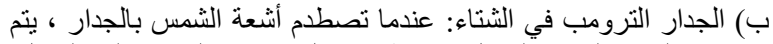

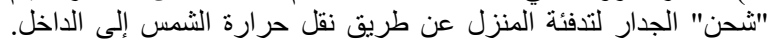

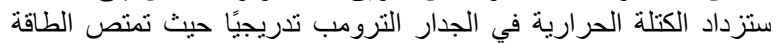

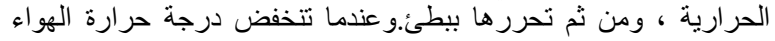

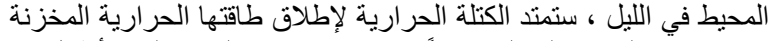

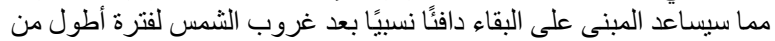

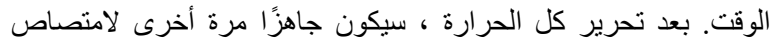

الحرارة في اليوم التالي.كما هو موضح في شئ شكل(3).
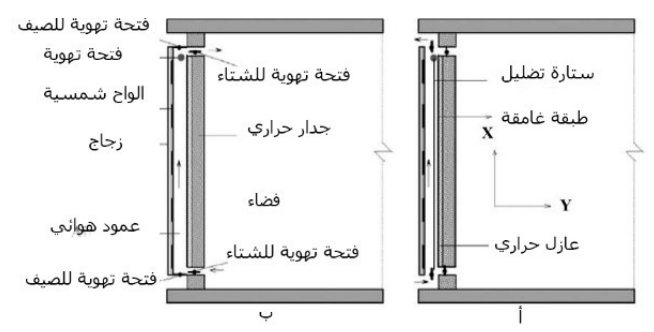

شكل 3:يوضح عمل الجدار الترومب في فصل الصيف(ب) وفي فصل الثتاء(أ),112].

\section{اعتبار ات رئيسة في تصميم الجدار الترومب}

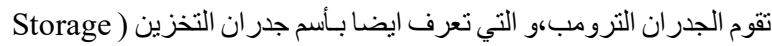

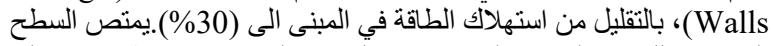

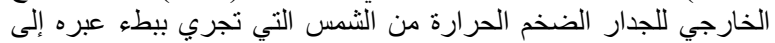

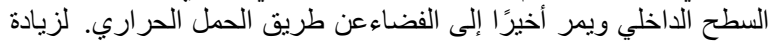

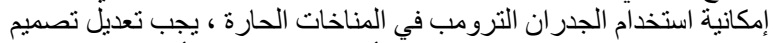

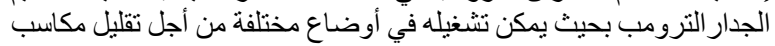

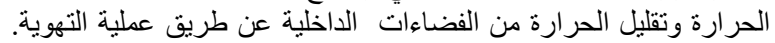

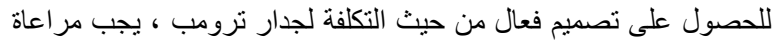

النقاط التالية: [1]

$$
\text { 1) تحديد نوع النظام اذا كان مهوى او غير مهوى }
$$

2) استخدام المر اوح: وجد أن الجدران ذات المروحة التي يتم التحكم فيها

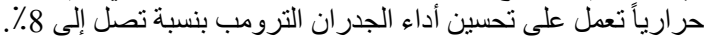

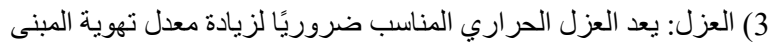

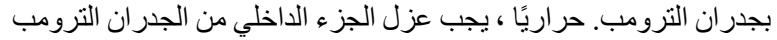

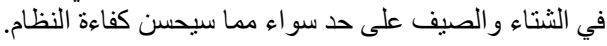

تطبيق الجدران الترومب في البيئة العمر انية العر اقية المعاصرة ذات المناخ

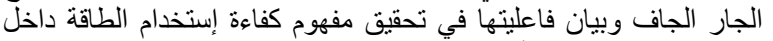

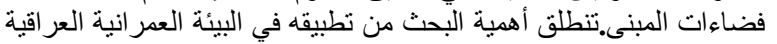

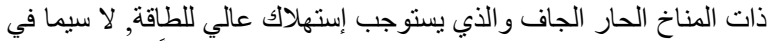

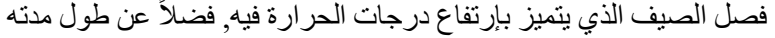

\section{3}

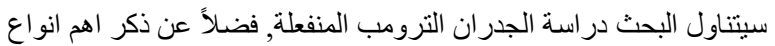

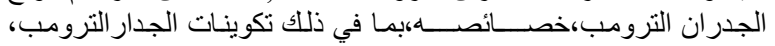

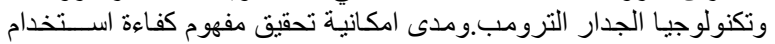

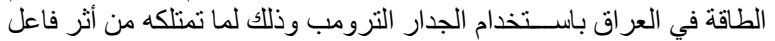

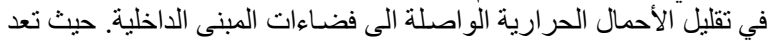

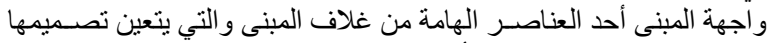

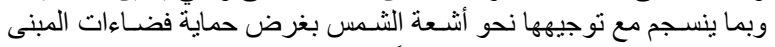
من الظروف المناخية و البيئية, علاوةً على تصميميها بإسلوب مستدام ينسجم مع المنطقة المناخية المحيطة بالمبنى.

4. عناصر ومكونات الجدار الترومب

1) جدار حجري بسماكة( 10 - 41 سم) مطلي بلون غامق في بمت فئص الحرارة

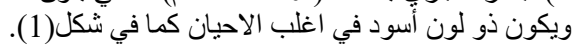

2) مغطى بزجاج معزول (زجاج مزدوج) أو زجاج منخفض الانبعاث

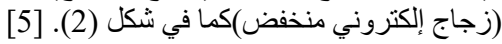

3) يتم وضع الزجاج على مسافة 2 - 5 سم بعيدًا عن جدار البناء لتوفير فجوة

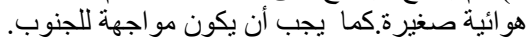

4) بالنسبة للمنازل الو اقعة في الجانب الجنوبي من خط الاستو اء ، يجب تثبيت

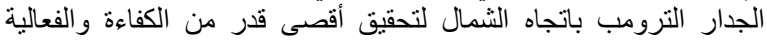
القصوى ، حيث أن هذا هو الجانب الذي سيتعرض لأشعة الثمس الأكثر.

5) زجاج نقل عالي يعزز المكاسب الثمسية للجدار. • الطبقة الزجاجية تمنع

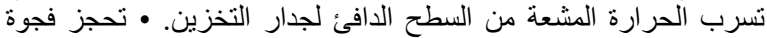
الهو اء الحرارة التي يشعها الجدار ، مما يؤدي إلى مزيد من التسخين لـن لسطح

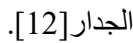

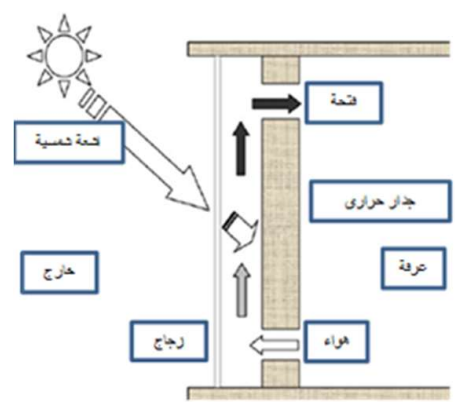

شكل 1:يوضح مكونات جدار ترومب,[5].

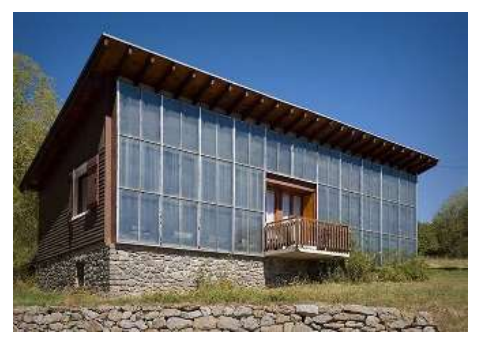

شكل 2:يوضح شكل جدار نرومب من الخارج,[5]. 


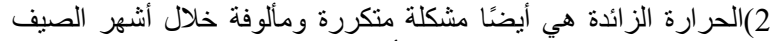

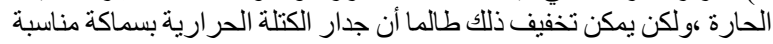
لامتصاص الحر ارة الز ائدة.

3) الجدران الترومب ذات مقاومة حر ارية منخفضة. خلال الليل أو فترات

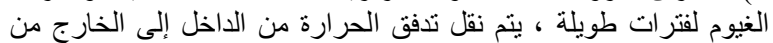

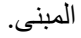

4) تعاني الجدران الترومب من ظاهرة السيفون الحرارية العكسية حيث

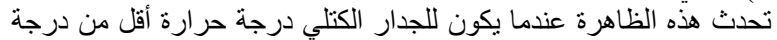

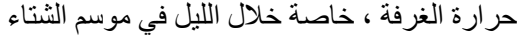

5) في الجدران الترومب يستمر نقل الحرارة دائمًا بشكل غير مؤكد. لا يمكن التنبؤ بكمية الحر ارة المكتسبة بسبب التغير ات في كثافة الطاقة الثمسية

6) الجدران الترومب ليست جميلة بما فيه الكفاية ، و القيمة الجمالية للجدران مشكوك فيها.

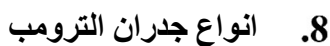

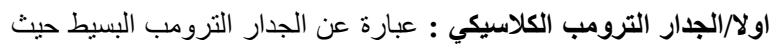

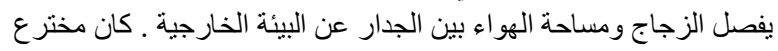

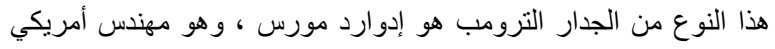

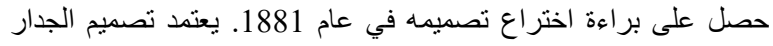

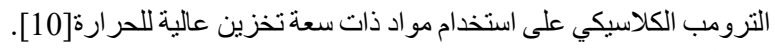

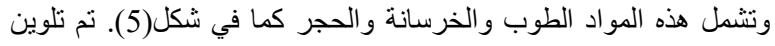

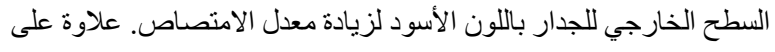

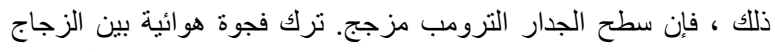

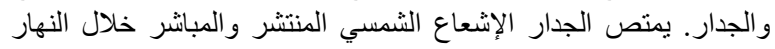

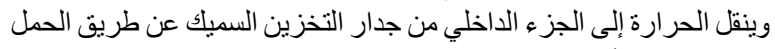

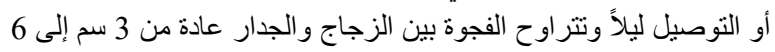

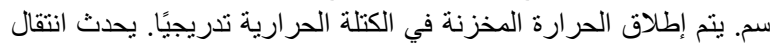

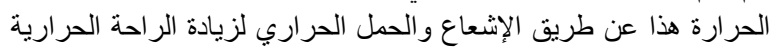

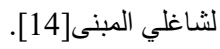

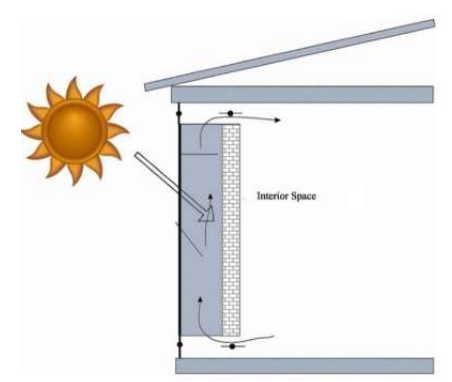

$$
\text { شكل 5:يوضح جدار ترومب الاكلاسيكي, [10]. }
$$

ثانيا/الجدار الترومب المتعرج:احد انواع الجدران الترومب تم تصميمه للحد

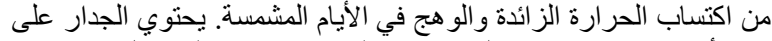

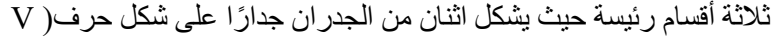

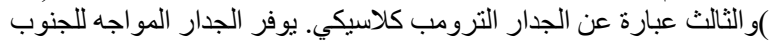

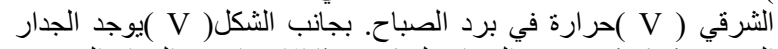

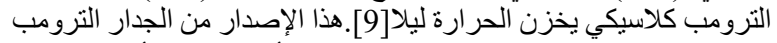

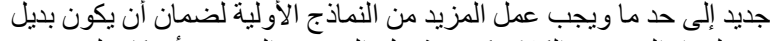

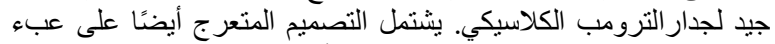

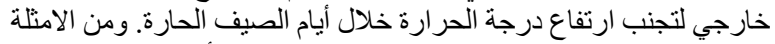

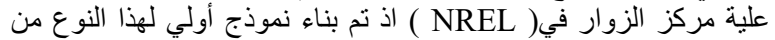

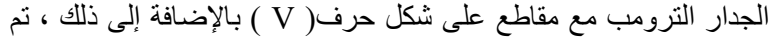

4) مساحة جدار: نسبة مساحة الجدار الترومب إلى إجمالي مساحة الجدار كمعيار مهم في تقييم كفائته [10]

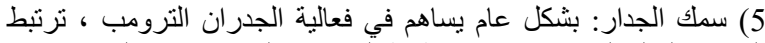

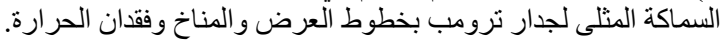

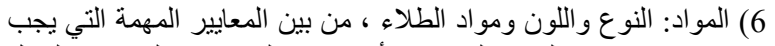

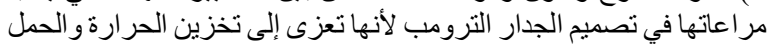

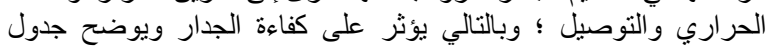
رقم(1) هذه المواصفات.

جدول 1:يوضح مواصفات الجدار الترومب, [10].

\begin{tabular}{|c|c|c|c|c|c|}
\hline اللجرارية الحلية & سلك & $\begin{array}{c}\text { ارتفار } \\
\text { الجذار } \\
\text { (م) }\end{array}$ & تظليل & مسباحة & توجيه \\
\hline 1.75 & $\begin{array}{c}0.25- \\
0.30\end{array}$ & $3-3.5$ & $\begin{array}{c}0.90- \\
0.95\end{array}$ & $\% 0-50$ & جنوب \\
\hline مواصفات & 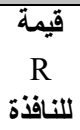 & الزجاج & الامتصاص الثمسي & والزجاج & الزجاج \\
\hline 1932 & 0.333 & 0.9 & 0.9 & $\begin{array}{c}0.15- \\
0.20\end{array}$ & $2-1$ \\
\hline
\end{tabular}

7. ايجابيات وسلبيات الجدار الترومب

ثُقدِم الجدران الترومب مستوئً عالي من الأداء و الخدمات, مما يجعله يحقق العديد من المز ايا, من أهمها : [5].

1) توفر هذه الجدران تدفئة مناسبة مقارنة بأنظمة التدفئة التقليدية الأخرى

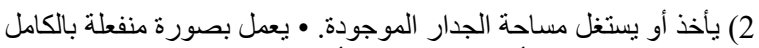

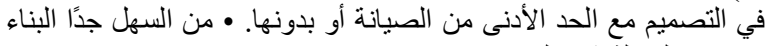
حتى مع العمالة شبه الماهرة.

3) يمكن لجدران الترومب أن تخفض فو اتير التدفئة بكميات كبيرة ويمكن أن

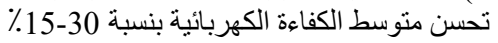

4) هو الأنسب في المناطق التي لديها موارد جيدة للطاقة الثمسية ومتطلبات كبيرة للتنفئة الثتوية.

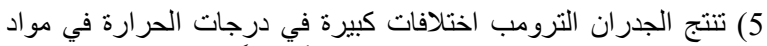

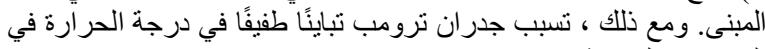

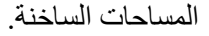

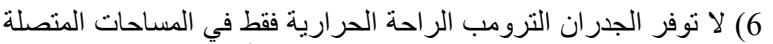
بجدار الترومب. و وانما توفر الر احة الحر ارية في الأماكن المجاورة.

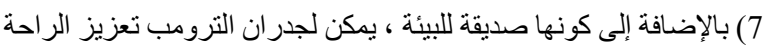
الحرارية وتوفير الطاقة حتى في المناطق القاحلة والصحر الهية الترية.

مع كل تصميم جديد ،هناك دائمًا بعض السلبيات جنبًا إلى جنب مع الايجابيات. فيما يلي سلبيات هذا النظام: [10-10].

1) قد يكون من المكلف تركيب الجدران الترومب. • يجب أن يتم تصميمه بعناية. 
الثمسية الزرقاء الداكنة أن تضيف جمالًا إلى المبنى وتزيد من جاذبية المبنى.

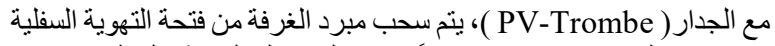

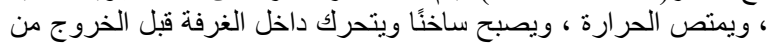

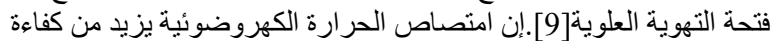

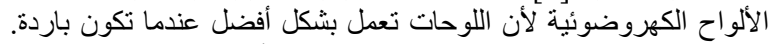

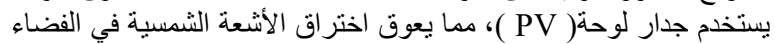

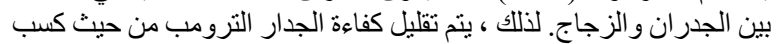

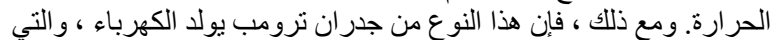
تعتبر مفيدة: [7].
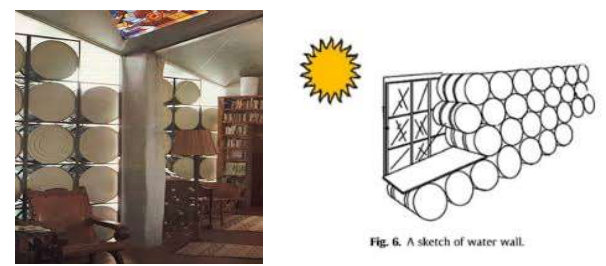

شكل 8:يوضح الجدار الترومب السائل, [14].

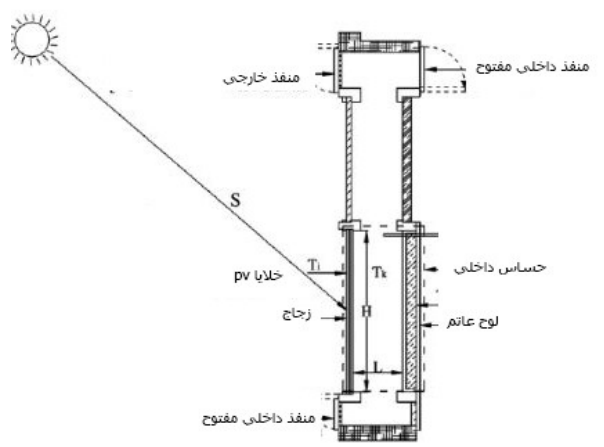

شكل 9:يوضح الجدار الترومب الكهروضوئي, [7].

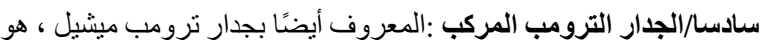

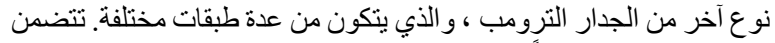

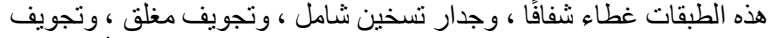

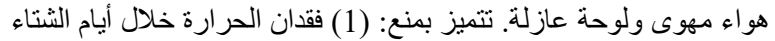

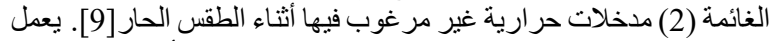

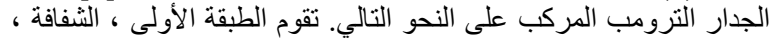

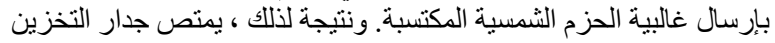

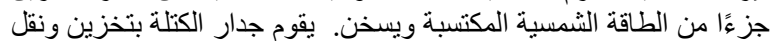

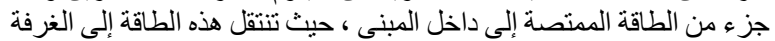

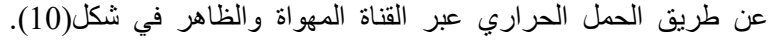

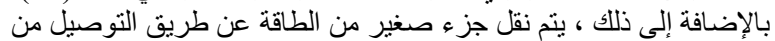

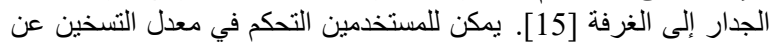

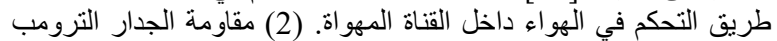

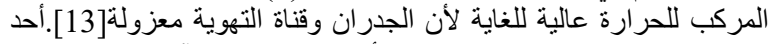

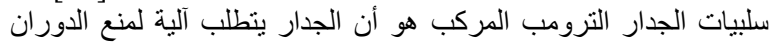

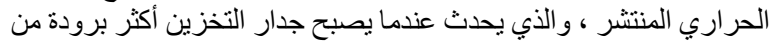

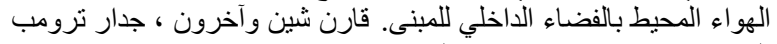

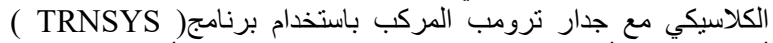

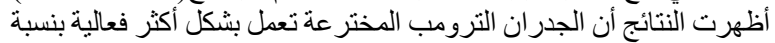
20-10٪ في المناخات القاسية ، مثل مناخ بوسطن [10].

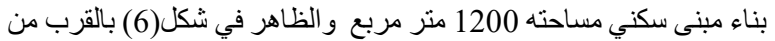

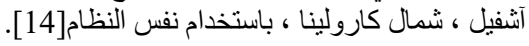
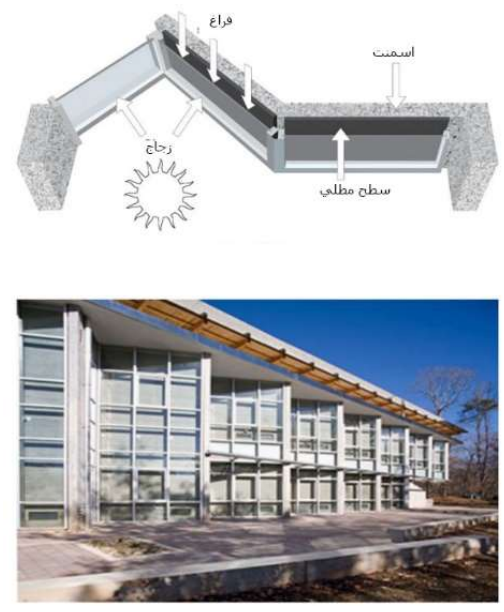

شكل 6:يوضح الجدار الترومب المتعرج و مركز الزوار في( NREL ), .[14]

ثالثا/الجدار الثمسي(Solar transwall): هو نوع نوع آخر من الجدار

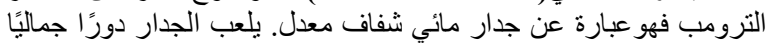

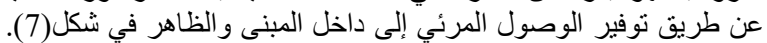

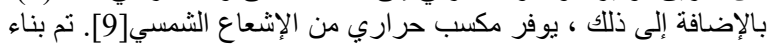

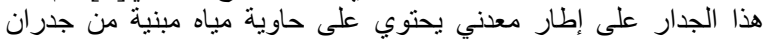

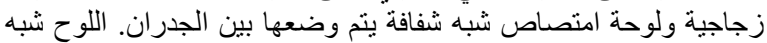

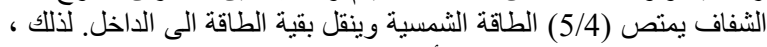

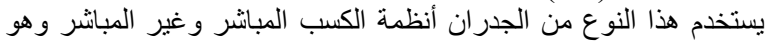

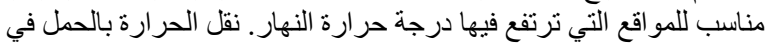

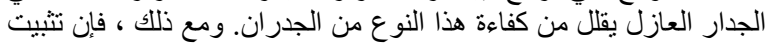

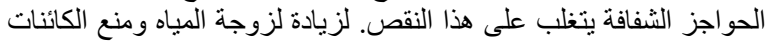

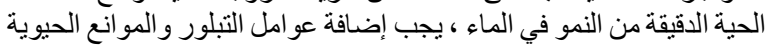

إلى الماء[2].

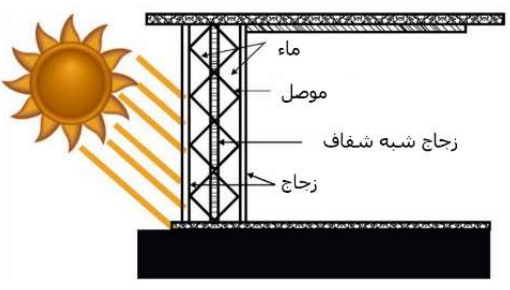

شكل 7:يوضح جدار ترومب الثمسي, [2].

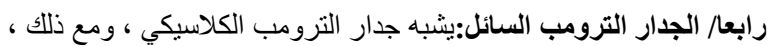

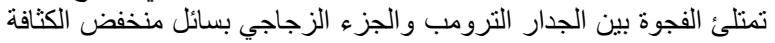

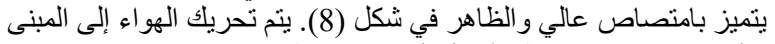

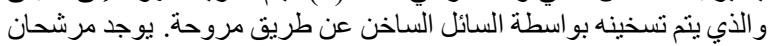

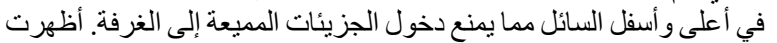

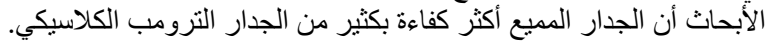

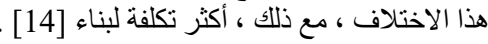

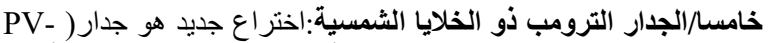
Trombe

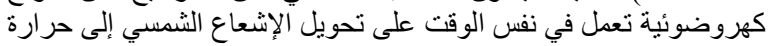

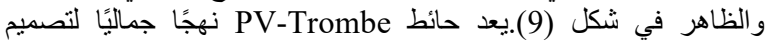

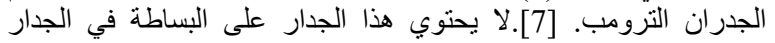
الترومب العادي ، حيث يغطى الزجاج بجدار كتلة سوداء ، لويمكن للخلايا 


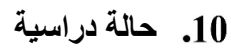

تم أخذ منزل نموذجي في بغداد في منطقة حطين المواجهة للجنوب كحالة

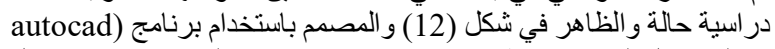

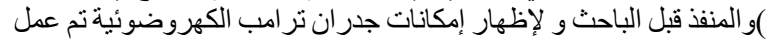

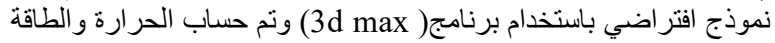

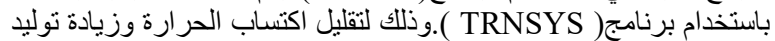

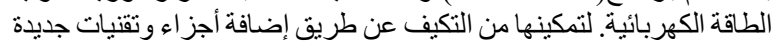

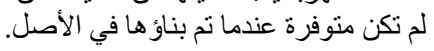

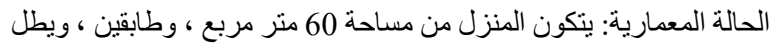

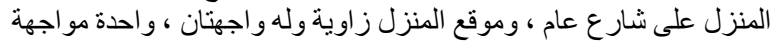

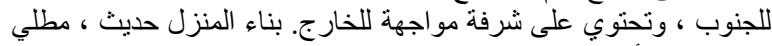

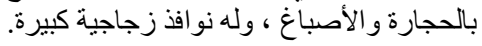
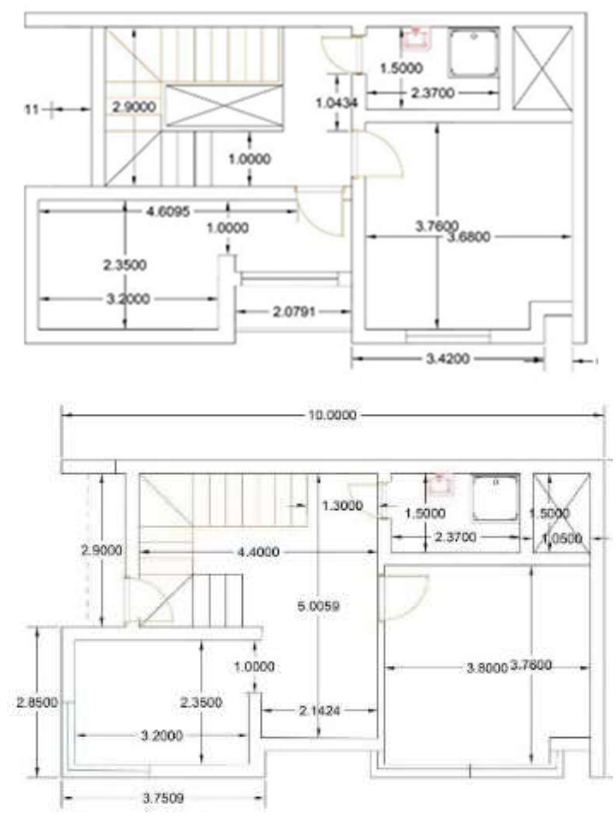

شكل12:مخطط منزل في منطقة حطين (مصدر : الباحث)

\section{1. تطبيق جوانب الجدار الترومب الكهروضوئي على منزل في منطقة حطين في بغداد}

وضع البحث العديد من المؤشرات المهمة المستخلصة من محاور الإطار

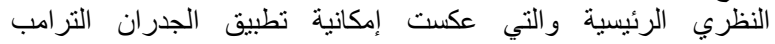

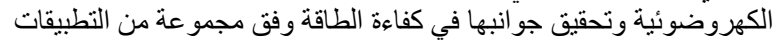

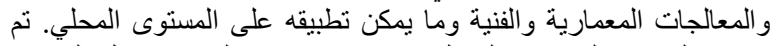

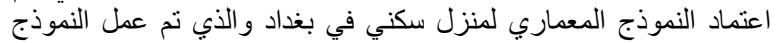

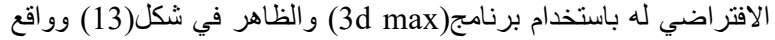

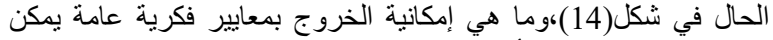

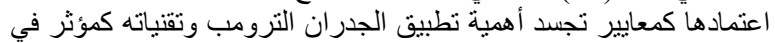
البناء التصميم. - nمعاير

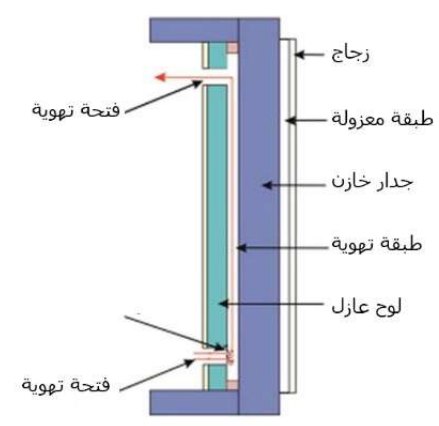

شكل 10:يوضح اجزاء الجدار الترومب المركب, [10].

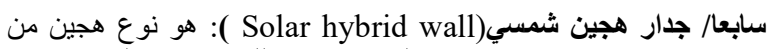

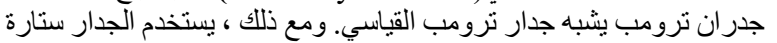

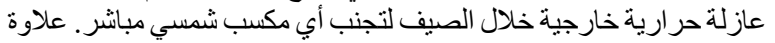

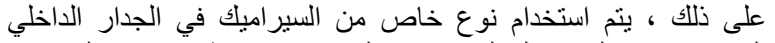

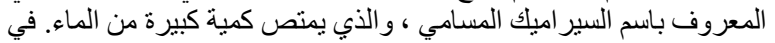

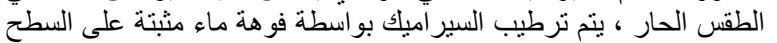

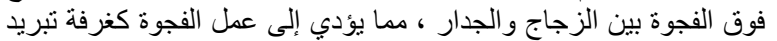

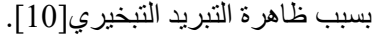

9 . الجدار الترومب وتحقيق مفهوك كفاءة استخدام الطاقة

مثل أي عنصر بناء آخر ، فإن الجدار الترومب هو مسألة تكاليف وفوائد ،

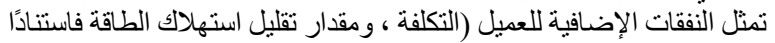

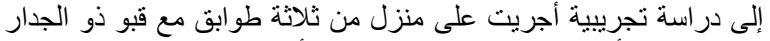

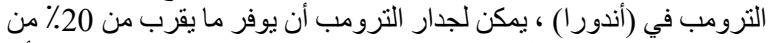

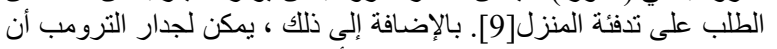

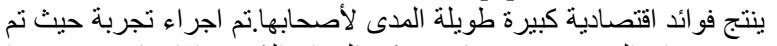

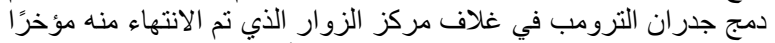

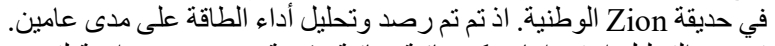

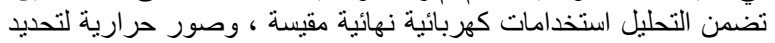

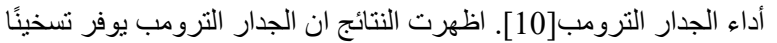

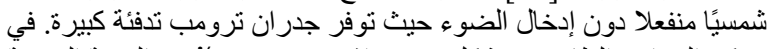

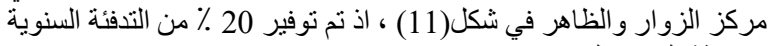

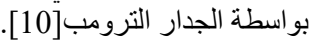

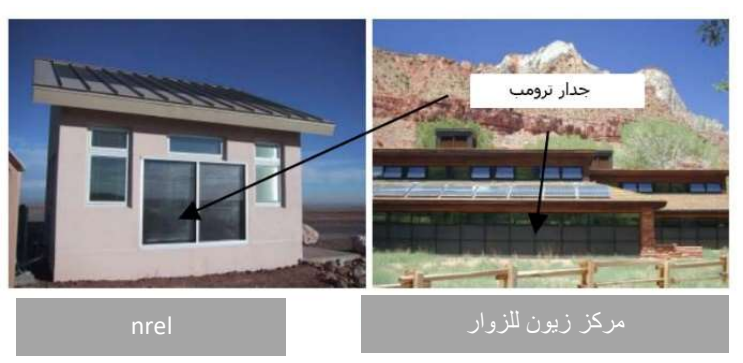

[9]11:بوضح مركز زوار

بناء على مما تقدم يمكن التوصل ان الجدران الترومب هو سمة معمارية

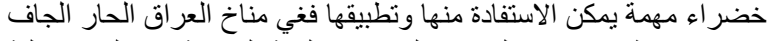

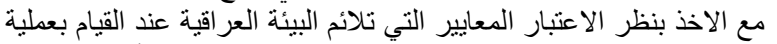

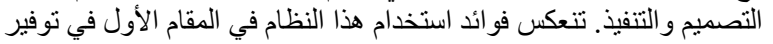

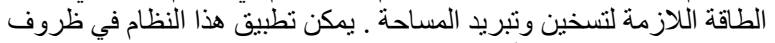

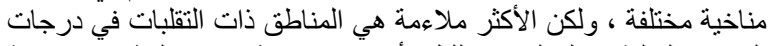

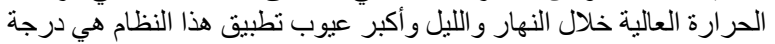
الكفاءة اعتمادًا على الظروف النار المناخية الخارجية. 


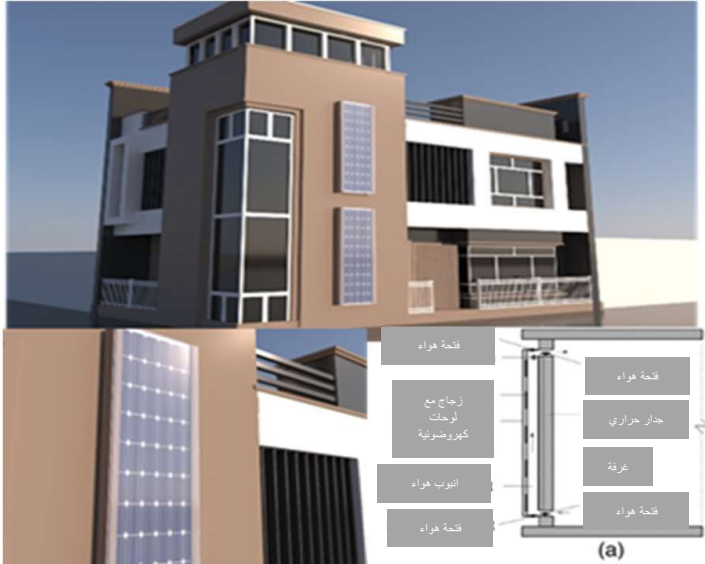

شكل 15:نماذج محاكاة منزل حطين المعدلة (مصدر :بو اسطة الباحث)

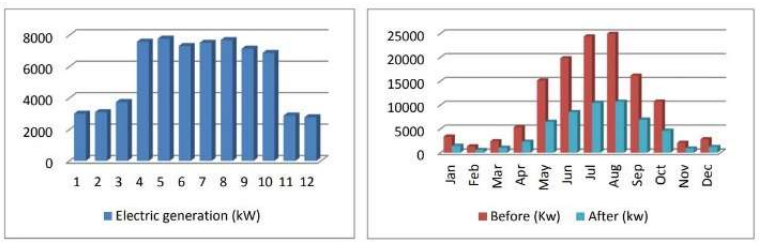

شكل 16:مقارنة استخدام نظام الجدار الترومب الكهروضوئي مقابل در اسة الحالة المرجعية لاستهلاكك الطاقة السنوي (المصدر : بو اسطة الباحث)

جدول 2: القيم الممكنة للإطار النظري (حسب الباحث)

\begin{tabular}{|c|c|c|c|c|c|c|}
\hline مكونات & جدار & مخرجات جدار & \multirow{2}{*}{\multicolumn{4}{|c|}{ نتائج جدار ترومب الكهروضوني }} \\
\hline المنزل & ترومب & ترومب & & & & \\
\hline & & & الاقتصاد & البيئة & الطاقة & \\
\hline جدران & 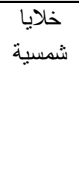 & 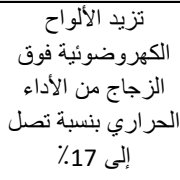 & & & & 1 \\
\hline شرفة & زجاج & الداخلية بمقدار 2.47 درجة الحر ارة & & & & 2 \\
\hline نافذة & 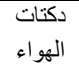 & كفاءة طاقة ازدادت & & & & 3 \\
\hline ابو اب & المنتارة & سبطرة على مناخ & & & & 4 \\
\hline
\end{tabular}

14. الاستنتاجات والتوصيات

14.1

كان لاستخدام الجدار الترومب الكهروضوئي فوائد كبيرة عكست نتائج

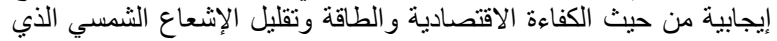

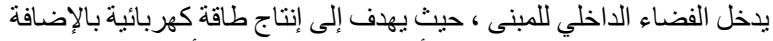
إلى العزل الحراري للمساحة.من أكثر النتائج المدهشة تأثير استخدام جدار بالئار

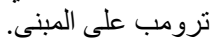

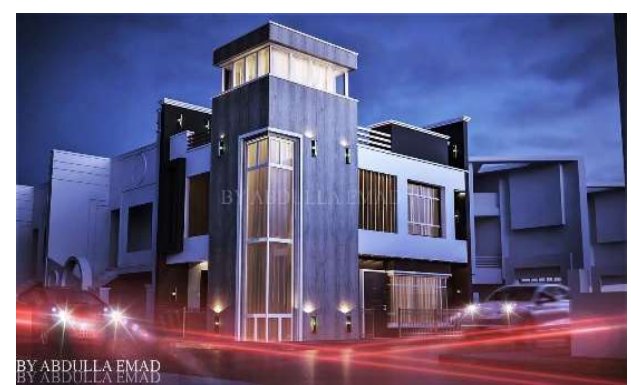

3d 13:تفاصيل المنزل المصمم من قبل الباحث باستخدام برنامج

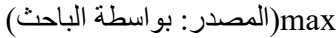

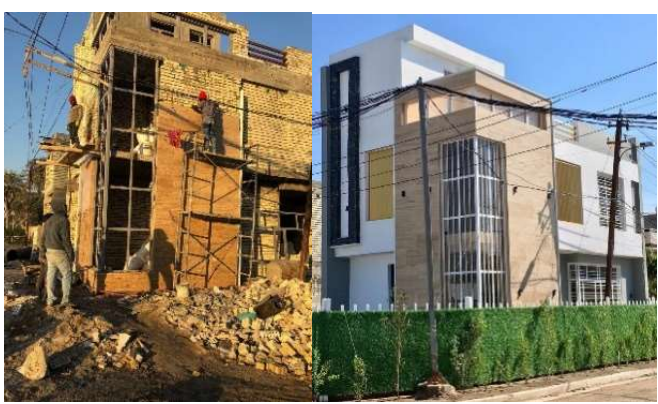

شكل 14:تفاصيل المنزل المصمم من قبل الباحث (المصدر: بو اسطة الباحث)

12. مخرجات تطبيق الجدار الترومب في العمارة

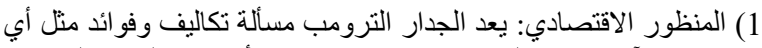

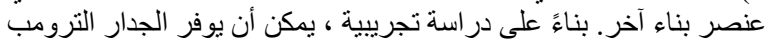

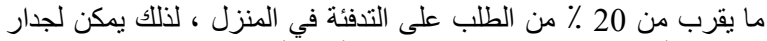

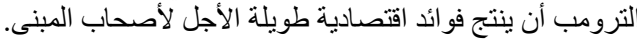

2) الاستدامة: تعد الجدران الترومب تقنية معمارية مستدامة للتدفئة و التهوية

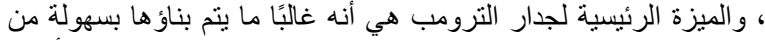

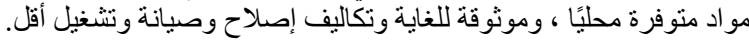

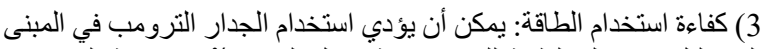

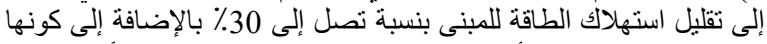

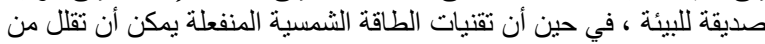
الطلب على التدفئة حتى 25\%

13.

بعد استعر اض عدة أنو اع من الجدران الترومب وذكر أهم العناصر و المعايير

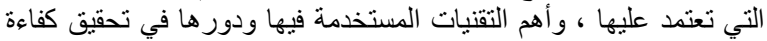

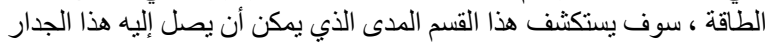

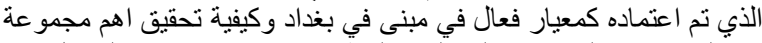

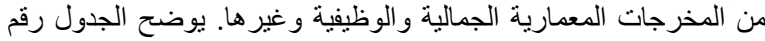

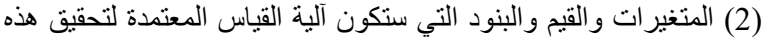

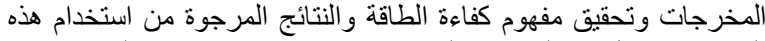

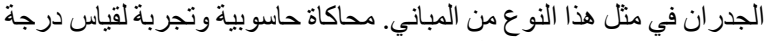

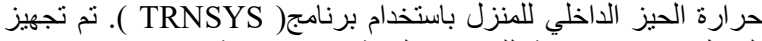

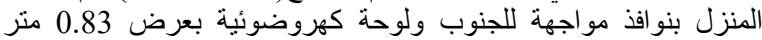

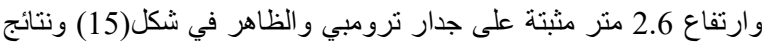

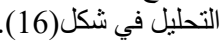


[4] Chan H-Y, Riffat SB, Zhu J. Review of passive solar heating and cooling technologies. Renewable and Sustainable Energy Reviews 2010

[5] Fakhreddine Abbassi, Narjes Dimassi, Leila Dehmani. Energetic study of a Trombe wall system under different Tunisian building configurations, Energy and Buildings 2014.

[6] Hordeski MF. New technologies for energy efficiency New York. The Fairmont Press; 2011.

[7] Jerzy Szyszka1, Janusz Kogut2, Izabela Skrzypczak. Selective Internal Heat Distribution in Modified Trombe Wall 2017.

[8] Kundakci Koyunbaba B, Yilmaz Z. The comparison of Trombe wall systems with single glass, double glass and PV panels. Renewable Energy 2012

[9] Llovera J, Potau X, Medrano M, Cabeza. Design and performance of energy-efficient solar residential house in Andorra 2010.

[10] Omidreza Saadatiann, K. Sopian, C.H. Lim, Nilofar Asim, M.Y. Sulaiman. Trombe walls: A review of opportunities and challenges in researchand development 2012

[11]Paul Torcellini and Shanti Pless. Trombe Walls in Low-energy Buildings: Practical Experiences. National Renewable Energy Laboratory 2004.

[12] Piyush Sharma, Sakshi Gupta. Passive Solar Technique Using Trombe Wall- A Sustainable Approach 2012

[13]Ryan D, Burek SAM. Experimental study of the influence of collector height on the steady state performance of a passive solar air heater 2010

[14] Sokol. Off the wall: Trombe walls at a visitor's center bask in the sunshine 2008 .

[15]Zalewski L, Joulin A, Lassue Sp, Dutil Y, Rousse. Experimental study of small-scale solar wall integrating phase change material 2012
النتائج الاقتصادية: لعب الجدار الترومب في هذه الدر اسة دورًا اقتصساديًا مهمًا

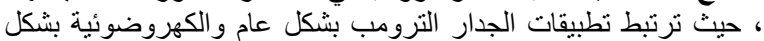

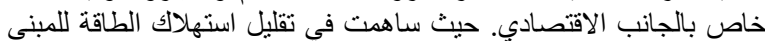

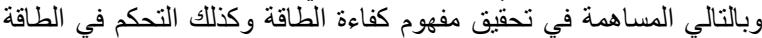

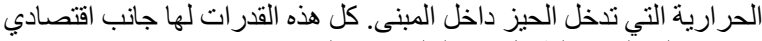

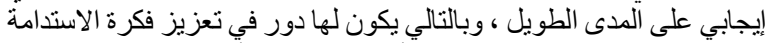

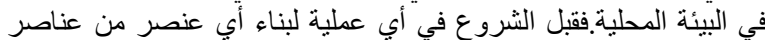

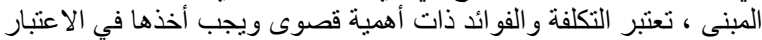

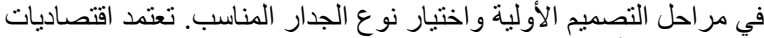

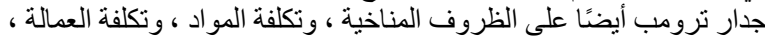

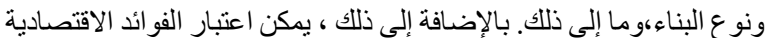

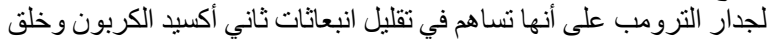
تدفق الهواء في المنطقة المجاورة.

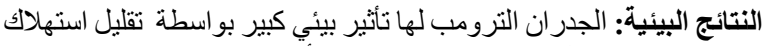

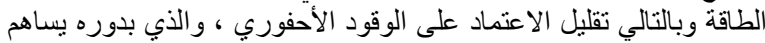

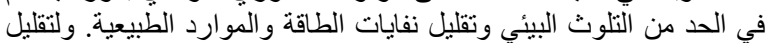

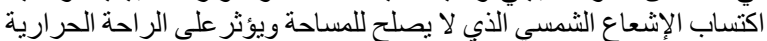
بالإضافة إلى زيادة الضوء الطبيعي وتقليل فقد الطاقة.

نتائج كفاءة الطاقة: أظهرت النتائج أن الجدار الترومب يوفر تدفئة شمسية

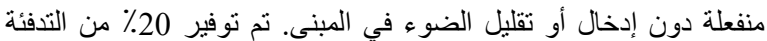

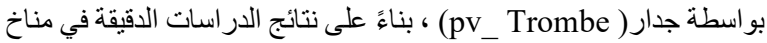
و اتجاه ومساحة المبنى.وخلصت الدر اسة إلى أنه يمكن نوفير 37٪ من التان التكلفة الإجمالية باستخدام هذا النظام. 14.2

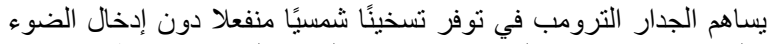

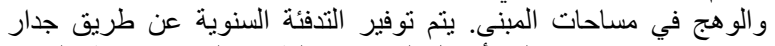

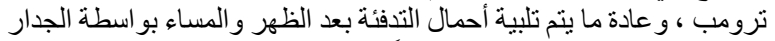

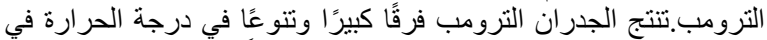

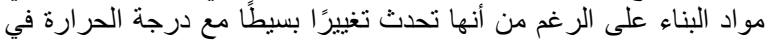

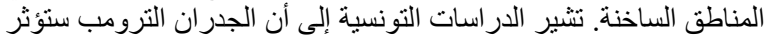

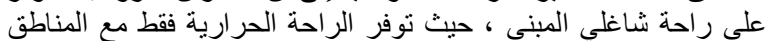

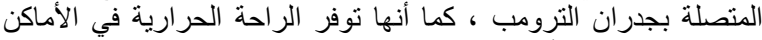

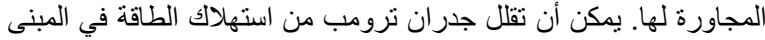

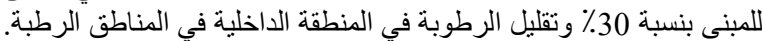

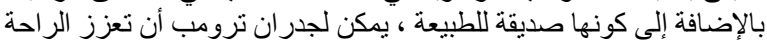

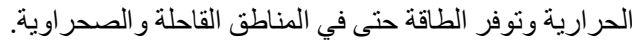

المصادر

[1] Ali M, Othman, Adnan I. O. Zaid. Utilization of the Trombe Wall in Hot Climate Regions: Jordan as a Case Study 2016.

[2] Al-Karaghouli A, Kazmerski LL. Optimization and life-cycle cost of health clinic PV system for a rural area in southern Iraq using HOMER software. Solar Energy 2010

[3] Al-Karaghouli A, Kazmerski. Optimization and life-cycle cost of health clinic PV system for a rural area in southern Iraq using HOMER software 2010. 


\title{
Adoption of the Trombe Walls as an Environmentally Sustainable Technology To Achieve The Concept Of Energy Efficiency In Hot Arid Regions
}

\author{
Abdullah Emad Aliwi ${ }^{*}$, Ghada Mohammed Ismael Abdul Razzaq Kamoona ${ }^{2}$ \\ ${ }^{1}$ Department of Architecture,University of Baghdad, Baghdad, Iraq, arch.abd.emad.94@gmail.com \\ ${ }^{2}$ Department of Architecture,University of Baghdad, Baghdad, Iraq, g.kamoona@coeng.uobaghdad.edu.iq \\ *Corresponding author: Abdallah Emad Aliwi, arch.abd.emad.94@gmail.com \\ Published online: 31 December 2020
}

Abstract- Trombe walls are one of the sustainable technologies because of their importance and effective rol in interior spaces by making use of solar energy to control the temperature of the interior spaces and thus achieve thermal comfort without the need to use cooling devices to reduce the energy used, the Trombe wall is an important element in solar design Passive. To save heat in indoor spaces, the main advantage of trombe walls is that they can be constructed virtually from locally available materials. These walls also reduce the need for heating and cooling the building. It is even considered a vital technology for sustainable architecture that effectively contributes to hot-arid environments like Iraq. The research will discuss the most important types of Trombe Walls, the characteristics of Trombe Walls, including the Trombe Wall formations, and the Trombe Wall technology, and the feasibility of realizing the concept of energy efficiency in Iraq using the trombe wall.

Keywords - sustainability, thermal comfort, solar energy, trombe wall. 